\title{
Towards a lightweight middleware for mobile multimedia communication awareness
}

\author{
Almudena Díaz \\ Departamento de Lenguajes y Ciencias de la \\ Computación \\ University of Málaga \\ Málaga, Spain \\ almudiaz@lcc.uma.es
}

\author{
Pedro Merino \\ Departamento de Lenguajes y Ciencias de la \\ Computación \\ University of Málaga \\ Málaga, Spain \\ pedro@lcc.uma.es
}

\begin{abstract}
The quality of service requirements for multimedia applications are well-known and have been widely discussed in the literature over the years. Though, the deployment of new radio access technologies and multimedia services over the new heterogeneous networks have given rise to new and challenging issues and, in this new context, improved performance of such applications is essential for the success of the next generation of mobile networks.

These new issues are being tackled from several points of view. In this paper we present a new approach for mobile device centres, based on providing the application level with a seamless communication adaptation to network issues. Due to the changeable nature of wireless network we have focussed on using information from lower levels to make the application level more adaptable. In other words, we propose to deploy a lightweight middleware based on cross-layer protocols for accessing lower layers parameters in order to provide adaptive techniques to the application level.
\end{abstract}

\section{Categories and Subject Descriptors}

C.2.1 [Computer-Communication Networks]: Network Architecture and Design-Wireless Communications; C.2.3 [Computer-Communication Networks]: Network Operations-network management, network monitoring

\section{General Terms}

Performance, measurement, management

\section{Keywords}

Middleware, mobile devices, adaptive techniques, cross-layer communications protocols, multimedia applications
Mobimedia 2008 July 7-9, 2008, Oulu, Finland. Copyright 2008 ICST ISBN 978-963-9799-25-7 DOI 10.4108/ICST.MOBIMEDIA2008.4023

\section{INTRODUCTION}

Moving multimedia services to the mobile environment requires adapting them to constraints such as network heterogeneity. As can be seen in figure 1, B3G systems (Beyond $3 \mathrm{G}$ ) are expected to be based on the integration of different technologies, such as $3 \mathrm{G}$ enhancements and WLAN. Vertical handover between these technologies is very important for efficient evolution to all-IP networks.

Another factor to take into account is the importance of vehicular scenarios in wireless networks. In such scenarios, radio conditions are very changeable and in order to achieve permanent application connectivity it is necessary to provide an effective mechanism for selecting the most appropriate radio technology and adapting the application configurations to it.

Due to the complexity of the scenario depicted, we propose implementing middleware which will assist new mobile multimedia applications to adap to constantly changing environment, with the objective of maintaining the optimal operation of applications.

Before moving on to the design the middleware, we have developed a tool named SymPA, which allows us to monitor low level communication parameters. With the data collected, we are able to characterize communication protocols behaviour over cellular networks and also to detect strategic parameters, thereby facilitating a seamless adaptation process in networking issues in wireless networks.

In previous works [6][7] we have used this tool for analyzing the performance of multimedia services over cellular networks. The knowledge acquired at this stage has allowed us to propose a new middleware centred on solving problems related with real mobility issues on cellular networks. Optimizing the quality of service perceived by users is the main objective of this middleware.

\subsection{Motivation}

The Third Generation Partnership Project (3GPP) has developed a series of specifications for a transparent end-toend packet-switched streaming service (PSS) which provides a framework for Internet Protocol (IP) based streaming applications in $3 \mathrm{G}$ networks. The technical specification for protocols and codecs [2] proposes the implementation of a 3GPP-Link-Char header field for enabling PSS clients to report the link characteristics of the radio interface to the PSS server. The specification also contemplates the implementation of adaptation and Quality of Experience (QoE) headers. These contain metrics and measurements collected 


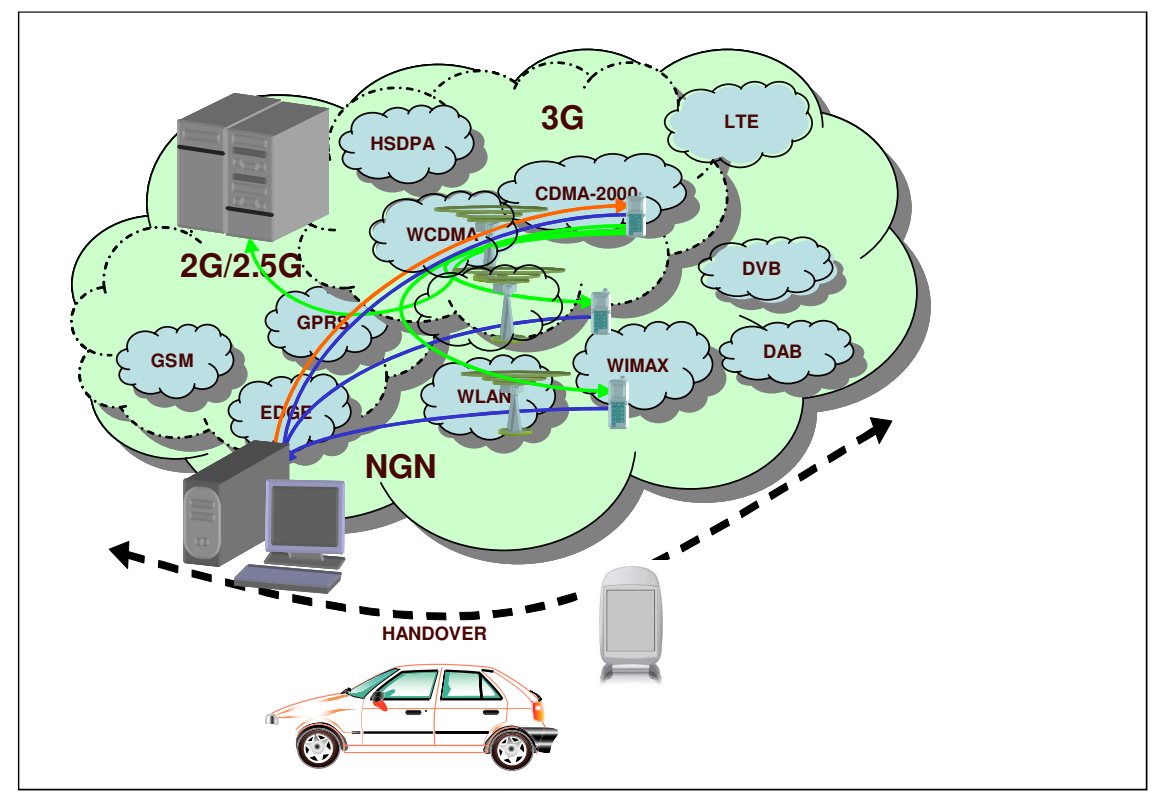

Figure 1: Application scenario

\begin{tabular}{|l|l|l|l|}
\hline \multicolumn{3}{|c|}{ Conversational Multimedia Application } \\
\hline Audio & Video & Text & \multirow{2}{*}{ RTCP } \\
\cline { 1 - 1 } \multicolumn{2}{|c|}{ Payload formats } \\
\cline { 1 - 1 } RTP \\
\hline \multicolumn{2}{|c|}{ UDP } \\
\hline \multicolumn{2}{|c|}{ IP } \\
\hline
\end{tabular}

Figure 2: User plane protocol stack for 3G packet switched conversational multimedia terminal

on the client side for sending feedback information to the server, and have been included in the specification to adapt transmission and content rates to the available network resources.

For the implementation of these new headers, applications not only need to manage information at the application layer but also need to access the underlying layer data. In the case of QoE headers, some of the parameters included are rebuffering, lost packets and jitter metrics. The first of these parameters can be easily reported by the client, but lost packet and jitter measurements require the implementation of new functionalities at the application level.

The middleware objective is to implement these new functionalities and to supply them to application level. The advantages of this approach are twofold:

- Application development is less complex.

- It allows the reuse of adaptive techniques from different multimedia applications, it being necessary only to adjust the performance requirements demanded for each multimedia application.
Also, to ensure, flexible functionality and to make the proposal useful the middleware not only centres on providing the next generation multimedia applications with the different adaptive techniques proposed by standardization organism but it will allow developers to implement their own adaptive techniques for their new applications and protocols.

\subsection{Why lightweight...?}

Mobile phones are devices with limited processing power and memory capacity. Nevertheless the main functionality of mobile phones is be able to be in contact at all times. A mobile phone must be operative during long periods of time and interruptions due to software errors are not admissible from the point of view of mobile users. Therefore it is not appropriate to overload such a system with a heavy software consuming resources which are vital for carrying out primary functions.

\section{RELATED WORKS}

In [11] the advantages of applying cross-layer techniques to wireless protocol stacks, are analyzed. This paper presents the advantages of communicating the application QoS needs to lower layers. In particular, the interaction with the physical and the link layer is considered crucial for improving application performance. Also some interesting use cases are provided. They contemplate the possibility of providing data to the users from lower layers data, in order for them to be able to decide on the number and the profile of the applications to be executed. Finally they propose a complex cross-layer architecture which involves numerous changes in comparison to traditional approaches.

In the literature there are other proposals about different kinds of mobile middlewares which focus on context management [5] [13], power management [3] or sensor and grid computing [9]. Fuego [12] is a mobile middleware centred on distributed applications, and it provides efficient services for data synchronization, based on XML, and data commu- 


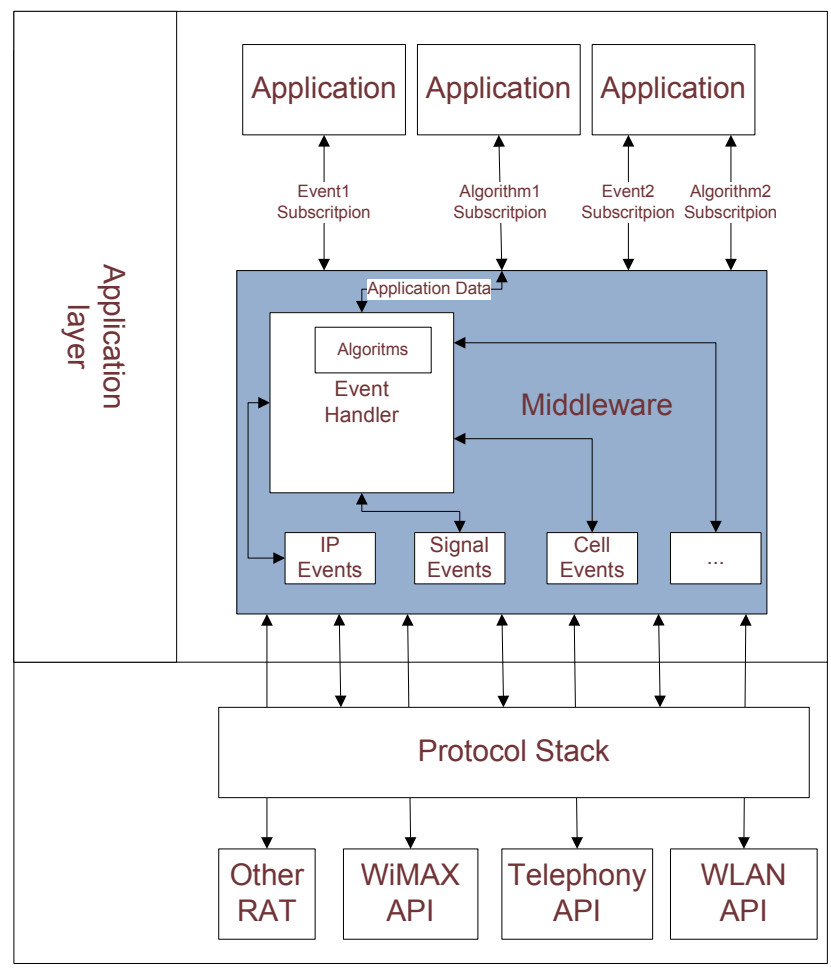

Figure 3: Event driven lightweight middleware

nications, based on SOAP and content-based routing.

We have focused our initial proposal on the optimization of multimedia applications with real time constraints, which are the most sensitive applications to the variable nature of wireless networks. We propose a more realistic approach based on a lightweight middleware, which operates at the application level, and on cross-layer communication protocols which are able to coexist with the traditional architecture of communication protocol stack.

\section{LESSONS LEARNED FROM PREVIOUS WORKS}

Because of the sophistication of new radio access technologies, in current wireless networks there are a lot of new issues which are short-lived and difficult to reproduce, and have revealed the lack of adequate testing and analysis for applications used in this type of environment [4]. Multimedia development needs the assistance of new tools to deal with these issues. With this objective in mind we have developed a new tool, named SymPA [8] to measure and analyze the behaviour of IP-based services and applications, from the point of view of end users.

The performance evaluation of multimedia applications is vital for detecting the erratic behaviour of mobile networks and applications. In previous works [6][7] we have analyzed the performance of video streaming traffic using cellular networks in real scenarios. The acquired understanding of mobility issues from the application level point of view allows us to design a middleware with appropriate functionalities from the application developers point of view.

In particular we have studied the impact of mobility is- sues in the performance of video streaming service. During trials we have used Real Time Protocol (RTP) as transport protocol. This is the protocol proposed by the ThirdGeneration Partnership Project (3GPP) for mobile video streaming over packet switched networks [2] and also for future services based on IP Multimedia Subsystem (IMS)[1]. Figure 2 shows the protocol stack proposed by $3 \mathrm{GPP}$ for the deployment of next generation multimedia services based on IMS. Hence, results obtained can be extrapolated to the performance of future services based on such protocol stacks.

For implementing the middleware functionality of the middleware three important issues have been identified which need to be addressed: jitter, packet losses and bandwidth.

During experimental trials a correlation was observed between packet size and jitter; higher jitter was obtained for higher packet size. Therefore one of the functionalities identified to be delegated in the middleware is the "packetization" of RTP content to make packet size suitable for the propagation characteristics of the radio technology being used.

Packet loss is one of the most restrictive parameters for multimedia applications. During trials, packet loss has appeared in vehicular scenarios as a result of low RSSI levels and during cell changes.

In this case we need on the one hand to implement predictive algorithms based on the RSSI level to anticipate packet losses and also cell changes. On the other hand, using these predictions, we need to implement dynamic techniques for dimensioning the internal buffers size of the middleware. These internal buffers will be used for managing the application data traffic.

The last functionality identified is the implementation of 
techniques for measuring the available bandwidth. The bandwidth available in wireless networks is very changeable, so the middleware must monitor bandwidth use to keep it under control. The use of internal buffers is also necessary for implementing this functionality.

For correct dimensioning of internal buffers, the middleware needs to take into account the delay constraints of each application.

\section{IMPLEMENTATION ISSUES}

One characteristic differentiating this approach from others is that specific objectives detected in previous work have allowed us to avoid ambiguities in the middleware approach, and to centre our efforts on real problems.

Due to the diversity of operating systems and platforms (Symbian OS, Windows Mobile, Linux, J2ME, Brew, Android, iPhone, etc) available in the mobile devices market, the middleware will be based on implementing a set of crosslayer protocols for accessing to low level data. This is the approach followed by JXTA, a language and platform independent set of protocols for peer-to-peer networking, developed by Sun.

The middleware proposed in this paper is not incompatible with others middlewares such as MIH [10]. It has been designed to obtain low level information for making it available to the application level underlaying events, so that the application is able to modify its behaviour.

For future extensions of the middleware, the event oriented implementation proposed in figure 3 provides a flexible and scalable architecture which will allow easy integration of new adaptation techniques, protocols and interpretability with other middlewares. To maintain the QoS parameters in line with reference values provided for standard specifications, protocols will be configured with these values. Event oriented implementation also provides and efficient power consumption.

Middleware can be used by applications in two different ways. Firstly it directly supplies adaptative algorithms to the application level. Secondly, in the operation mode based on even propagation, the middleware provides an application with the information needed for implementing adaptive techniques. This is a very flexible mode which allows new and innovative techniques to be developed.

\section{CONCLUSIONS}

Providing a middleware for communication awareness is essential for supportting the growth of the mobile Internet. We have used a software tool for protocol analysis and performance measurement, which runs on smart phones, to support the design of middleware functionality. Prior to the design of the middleware we identified the source of multimedia communication problems, obtaining real end-to-end information from the subscriber perspective. Finally, we have provided an event-driven lightweight middleware design and have identified the three main functionalities of the middleware.

\section{ACKNOWLEDGMENTS}

This work has been funded both by the Spanish government sponsored project TIN 2005-09405-C02-01 and by project TIC 03131 funded by the Autonomous Community of Andalusia.

\section{REFERENCES}

[1] 3GPP. Technical specification group services and system aspects; packet switched conversational multimedia applications; transport protocols (release 7). TS 26.236, 3GPP, 2008.

[2] 3GPP. Technical specification group services and system aspects; transparent end-to-end packet-switched streaming service (pss); protocols and codecs (release 7). TS 26.234, 3GPP, 2008.

[3] H. S. Ashwini, A. Thawani, and Y. N. Srikant. Middleware for efficient power management in mobile devices. In Mobility '06: Proceedings of the 3rd international conference on Mobile technology, applications $\mathcal{E}$ systems, page 49, New York, NY, USA, 2006. ACM.

[4] K. A. Brown. Impact of wireless communication on multimedia application performance. In Proc. SPIE Vol. 3528, p. 566-573, Multimedia Systems and Applications.

[5] R. C. A. da Rocha and M. Endler. Middleware: Context management in heterogeneous, evolving ubiquitous environments. IEEE Distributed Systems Online, 7(4):1, 2006.

[6] A. Diaz, A., P. Merino, L. Panizo, and A. Recio. Evaluating Video Streaming Over GPRS/UMTS Networks: A Practical Case. In Proc. VTC2007-Spring Vehicular Technology Conference IEEE 65th, pages 624-628, 2007.

[7] A. Diaz, P. Merino, L. Panizo, and A. M. Recio. Experimental analysis of peer-to-peer streaming in cellular networks. In Proc. 21st International Conference on Advanced Information Networking and Applications AINA '07, pages 784-791, 21-23 May 2007.

[8] A. Diaz, P. Merino, and F. J. Rivas. Customer-centric measurements on mobile phones. In Proceedings on 12th IEEE International Symposium on Consumer Electronics. ICSE 2008. Vilamoura, Portugal. 14-16 April, page 4, Los Alamitos, CA, Apr. 2008. IEEE Computer Society.

[9] H. Franke, F. Koch, C. Rolim, C. Westphall, and D. Balen. Grid-m: Middleware to integrate mobile devices, sensors and grid computing. In Proc. Third International Conference on Wireless and Mobile Communications ICWMC '07, pages 19-19, 2007.

[10] IEEE. Draft standard for local and metropolitan area networks: Media independent handover services. Unapproved Draft Std P802.21/D10.0 802.21, IEEE, 2008.

[11] V. Raisinghani and S. Iyer. Cross layer design optimization in wireless protocols stacks. Computer Communications, 27(8):720-725, May 2004.

[12] S. Tarkoma, J. Kangasharju, T. Lindholm, and K. Raatikainen. Fuego: Experiences with mobile data communication and synchronization. In 17th Annual IEEE International Symposium on Personal, Indoor and Mobile Radio Communications (PIMRC).

[13] S. S. Yau and F. Karim. A context-sensitive middleware for dynamic integration of mobile devices with network infrastructures. J. Parallel Distrib. Comput., 64(2):301-317, 2004. 